\title{
Impact of Marijuana Use on Self-Rated Cognition in Young Adult Men and Women
}

\author{
Deirdre A. Conroy, Ph.D¹, Megan E. Kurth, MPH², Kirk J. Brower, MD¹, David R. Strong, \\ Ph. D ${ }^{3}$, and Michael D. Stein, MD ${ }^{2,4}$ \\ ${ }^{1}$ University of Michigan Addiction Research Center, Ann Arbor, MI 48109 \\ ${ }^{2}$ General Medicine Research Unit, Butler Hospital, Providence, RI, 02906 \\ ${ }^{3}$ Department of Family and Preventive Medicine, University of California, San Diego, La Jolla, CA, \\ 92093 \\ ${ }^{4}$ Warren Alpert School of Medicine of Brown University, Providence, RI, 02912
}

\section{Abstract}

Background and Objectives-Marijuana (MJ) is a widely used substance that has been shown to impair cognition in laboratory settings. There is a growing number of medical marijuana dispensaries and state policies permitting the use of MJ in the United States. This study is a naturalistic study that explores the association of same day marijuana use on self-rated cognition in young adult men and women.

\begin{abstract}
Methods-Forty-eight young adults (22F; mean age=22.3) participated. After a baseline assessment, participants made daily phone calls to study staff over the next three weeks. Cumulative minutes of MJ use in the last 24-hours were assessed. Demographic information were collected and self-ratings of cognitive impairment were assessed using six questions about areas of difficulty thinking each day.
\end{abstract}

\begin{abstract}
Results-There was a significant relationship between greater number of minutes of marijuana use and higher levels of self-rated cognitive difficulties $(b=0.004 ; \mathrm{SE}=0.001 ; \mathrm{p}<0.006)$. There was no main effect of gender $(b=1.0 ; \mathrm{SE}=0.81 ; \mathrm{p}<0.22)$. Planned evaluation of the interaction between gender and minutes of marijuana use was not significant statistically, suggesting a similar relationship between minutes of marijuana use and cognitive difficulties among women compared to men $(\mathrm{p}<0.54)$.
\end{abstract}

Conclusions and Scientific Significance-There is an association between current and heavy MJ use and self-perceived cognitive ability in both males and females. These findings reveal important information regarding one consequence of $\mathrm{MJ}$ use that has real-world meaning to young adult smokers.

Corresponding author: Deirdre A. Conroy, Ph.D. 4250 Plymouth Road Ann Arbor, MI 48109 Office: 734-232-0559 Fax: 734-998-7992 daconroy@umich.edu.

Declaration of Interest: The authors report no conflicts of interest. The authors alone are responsible for the content and writing of this paper. 


\section{Keywords}

marijuana; cognition; self-rated; sex differences; naturalistic

\section{INTRODUCTION}

Marijuana (MJ) is one of the most commonly used illicit drugs and its use in young adults is once again on the rise ${ }^{1}$. There are a growing number of medical MJ dispensaries and state policies permitting the use of MJ in the United States ${ }^{2}$. Given this increase in more lenient laws, there is a pressing need to better understand the consequences of MJ use, particularly those that are perceived by the user. This study explores impact of marijuana use on selfrated cognition and whether this differs between young adult men and women. Whether MJ affects cognition in females and males differently is an area that is still poorly understood. Animal research has shown that female rats are more sensitive to the effects of cannabinoids, due in part to the lower number of CB1 receptor binding sites in cerebral areas of females compared to males ${ }^{3}$. In addition, chronic exposure to THC has been shown to be associated with different emotional responses in male and female rats ${ }^{4,5}$. One of the first studies to show sex differences in MJ response in cognitive tasks in humans was by Pope et al $(1997)^{6}$. In that study, visuospatial memory tests were compared in males and females who were heavy and light MJ smokers. The study showed no significant differences between heavy and light smokers, but when females were analyzed separately from males, heavy smoking females had more difficulty remembering and made more errors than light smoking females ${ }^{6}$. Two recent reviews of the influence of sex on the effect of MJ use 7,8 highlight the influence of hormones on the sensitivity of females to MJ exposure and the need for the careful evaluation of potential differences in the effects of MJ on cognition among females. However, no study has systematically examined subjective differences in cognition between males and females.

Studies have shown a clear connection between the acute adverse effects of cannabis on cognition ${ }^{9}$, but this changes depending upon the frequency of use and length of abstinence ${ }^{10}$. Recent studies conducted on the effects of MJ on cognition in young adult users assess cognition well after the use of MJ. A typical measure for "recent MJ use" is in the last 4 weeks ${ }^{11-13}$. Another study on the effect of MJ use on cognition in a young adult population asked that participants refrain from MJ use for at least 12 hours before testing so as not to be high during the assessment ${ }^{14}$. Therefore, consideration of same day MJ use with self-rated cognition would be an important area of examination in studies of the effects of MJ on neurocognition. In the current study, we investigated how variations in MJ use influence self-rated cognition when assessed on a daily basis in a naturalistic setting. We hypothesized that greater levels of MJ use would be associated with lower self-ratings of cognition, daily functioning, and that females would report worse ratings than men. 


\section{METHODS}

\section{Participants}

Participants (26 M; 22F) between the ages of 18-30 were recruited between March 2012 and March 2013 through on-line advertisements in Craig's List and Facebook, looking for "persons 18-29 who drink alcohol or smoke marijuana." Approximately half of the participants had at least a high school education, were unemployed, or were students at the time of the study (Table 1). At screening, young adults who reported any MJ use in the past month were eligible. Because we were interested in trying to isolate the effect of MJ on selfperceived cognition, our exclusion criteria were: 1) no past month cocaine, opioid, benzodiazepine, or stimulant (methylphenidate, amphetamine, methamphetamine) use or positive urine toxicology for these substances prior to the baseline interview, 2) no more than once a month binge drinking (defined as 5 or more drinks within a 2-hour period for men, 4 or more drinks within a 2-hour period for women), 3) night shift work, 4) selfreported history of schizophrenia, bipolar disorder, or currently medicated attention deficit hyperactive disorder, 5) lack of stable housing, and 6) current (past 2 weeks) suicidal ideation, 7) past month use of sleep medication or antidepressants. We also recruited through Craig's List and Facebook, an age-matched group who reported no MJ use in the last month to complete a continuum of MJ use patterns. We included individuals with patterns of MJ use that ranged from no-use, non-daily, and daily use who provided reports of variability in daily functioning that could be related to reported levels of MJ use on use and non-use days.

A total of 646 persons were screened by phone for eligibility. Of these, 525 were ineligible due to recent binge drinking or other alcohol-related problems. An additional 44 were not eligible due to reporting one or more other exclusion criteria. These included (numbers reported are not mutually exclusive) use of other drugs $(n=22)$, mental disorder diagnosis (schizophrenia, bipolar disorder, currently medicated attention deficit hyperactive disorder) $(n=23)$, currently being treated for depression $(n=10)$, suicidality $(n=10)$, unstable housing $(n=4)$, and no phone $(n=1)$. Of the eligible participants, 6 could not be reached to schedule a baseline appointment, 2 refused participation, and 20 did not come in for their baseline appointment. Forty-nine agreed to participate in the study and were consented; and one person was not included in analyses due to substantial missing data $(\mathrm{N}=48)$.

\section{Measures}

All participants were administered a Structured clinical interview for DSM-IV - Patient version (SCID) ${ }^{15}$ at baseline to assess for marijuana dependence. Eligible participants were provided with a daily diary created by the investigators which included questions about past 24-hour substance use and self-rated cognitive impairment. Participants could provide a maximum of three weeks of daily diary data.

\section{MJ Use and Self-rated Cognition}

Last 24-hour MJ use was assessed at the daily calls. We used minutes as a proxy of time spent smoking. The subject estimated how many minutes they spent consuming/using/ smoking MJ and not the minutes they felt intoxicated. The instruction given to subjects was: 
How many minutes do you think you spent actually smoking marijuana?" The authors chose to use minutes of marijuana because it is very difficult to ascertain in the naturalistic setting exactly how much THC the participant is ingesting. To clarify this measure further, we made frequency of use in days (daily vs. non-daily use) and meeting a diagnosis of dependence as our secondary measures. In addition, cumulative minutes of marijuana use during four 6-hour blocks (midnight to 6am, 6am - noon, noon - 6pm, 6pm-midnight) were assessed. Self-rating of cognitive impairment was assessed using six questions from the cognition subscale of the Medical Outcome Study Measure (cognitive function) short-form general health survey about areas of difficulty thinking each day ${ }^{16}$. Research staff asked, "Yesterday, how much of the time did you: 1) have difficulty reasoning and solving problems (for example, making plans, making decisions, learning new things)?; 2) have difficulty doing activities involving concentration and thinking?; 3) become confused and start several actions at a time?; 4) forget, for example, things that happened recently, where you put things, appointments?; 5) have trouble keeping your attention on any activity for long?; 6) react slowly to things that were said or done? Six response options (scored 0-5) ranged from none of the time to all of the time. Responses were summed (possible range $0-30)$ and the internal consistency reliability from the first day of ratings for this measure was good (Cronbach's alpha $=0.85$ ). The internal consistency (Cronbach's alpha) of the cognitive problems instrument ranged from 0.84 - 0.92 across the evaluation days.

\section{Procedures}

Eligible individuals were asked to come in to Butler Hospital for informed consent and a baseline interview. This study was approved by the Institutional Review Boards of Butler Hospital and the University of Michigan. At baseline, participants received a 40-minute face-to-face interview to assess demographics, and marijuana use. Participants also provided urine samples for toxicological analysis to rule out recent drug use prior to this interview. Participants agreed to four in-person assessments over 3 weeks (baseline, and 1, 2, and 3 weeks) and to make daily 5-minute phone check-ins with research staff during the 3-week period. A urine sample was also collected at each assessment. All participants were asked to avoid binge drinking, drug use other than marijuana, and sleep medication for the duration of their study participation. It was explained that these behaviors would not affect participation, however, and they should report all drug and alcohol use accurately on their daily phone diary.

Daily Phone Contact-At baseline, participants chose a 90-minute window during which they could make a brief phone call to research staff every day for the next 3 weeks. They were asked to choose a time as close to their usual wake time as possible, and to have a diary filled out in advance, in order to make the phone call less burdensome. Participants were given paper copies of the diary, and completed their first diary with research staff at the baseline interview.

\section{Statistical Analysis}

We used maximum likelihood estimation of generalized linear mixed effects negative binomial regression models with random intercepts to model the repeated daily assessment of self-rated cognitive impairment and accommodate cases with any missing assessments. 
The negative binomial model allows modeling of the positively skewed count of cognitive impairments symptoms. All models included planned covariates of 1) time 2) education (Up to High School, Some College) , 3) current employment status (Unemployed, Part-time, Full-time) 4) gender 5) Baseline days of alcohol use and 6) a term reflecting the main effect of day-to-day variation in level of marijuana use (minutes/day). After the main effect of marijuana use was evaluated we added a planned interaction term (Marijuana use X Gender) to assess whether the strength of association was different for men and women. We conducted analysis on the 48 enrolled participants who provided baseline daily diary phone responses. All statistical analyses were completed using R Core Team (2013).

\section{RESULTS}

Table 1 lists demographic characteristics of men and women in this study. Daily diary data were available for a mean of $21.86(\mathrm{SD}=3.41)$ and 21.0 (2.79) of the targeted 22 days for women and men, respectively. No participants reported use of sleep medication and 7 women (31.8\%) and 9 men (34.6\%) reported at least one heavy drinking episode during the assessment period. Average age of first marijuana use was 15.8 years of age.

\section{Marijuana Use and Daily Cognitive Difficulty}

Marijuana users were separated into the following three categories: non-users $(n=9)$, daily MJ users ( $n=16)$ and non-daily MJ users ( $n=23)$. Non-users served as the control group for this sample. On each day of MJ use, participants were asked how many minutes they smoked MJ, and during which hours, the day divided into 4 six-hour quadrants. Daily smokers were defined as persons smoking MJ at least six days per week, and non-daily smokers were persons who smoked on at least 1 day in the past month. Non-users were participants who had not smoked MJ in the last month.

We observed a wide range of marijuana use with a median of 10 minutes per day for women (Interquartile range $=0-50$ ) and 15 minutes per day for men (Interquartile range $=0-39$ ) during the three week evaluation period. Our sample also included broad range of marijuana use frequency. Women reported using $\mathrm{MJ}$ on a median of $81 \%$ days (Interquartile range, $16 \%-100 \%$ ) and men reported using on $86 \%$ of observed days (Interquartile range, $18 \%-100 \%)$." A Spearman rank correlation between average minutes of MJ use during the three-week evaluation period and the percentage of days using MJ during that same period was rho $=0.88(\mathrm{p}<0.001)$.The correlation between average minutes use during the threeweek evaluation period and a baseline DSM-IV diagnosis of Cannabis Dependence was $\mathrm{r}=0.33$ ( $\mathrm{p}=0.10$ ). Although not significant statistically, those with a diagnosis of cannabis dependence reported an average of $41.98(\mathrm{SD}=32.83)$ minutes as compared to an average of $23.60(\mathrm{SD}=34.36)$ minutes among those without a diagnosis, a moderate effect size (Cohen's d=0.53).

Levels of daily self-rated cognitive difficulties were a mean of $3.11(\mathrm{SD}=4.66$; Interquartile range $=0-5)$ for women and $1.96(\mathrm{SD}=3.68$; Interquartile range $=0-2)$ for men. Evaluation of the main effects in a regression model (see Table 2) with adjustment for linear effect of time, level of education, current employment status, gender, baseline days of alcohol use and level of marijuana use (minutes/day), revealed that there was a significant relationship 
between greater number of minutes of marijuana use and higher levels of self-rated cognitive difficulties $(b=0.004 ; \mathrm{SE}=0.001 ; \mathrm{p}<0.006)$. There was no main effect of gender $(b=1.0 ; S E=0.81 ; p<0.22)$. Planned evaluation of the interaction between gender and minutes of marijuana use was not significant statistically, suggesting a similar relationship between minutes of marijuana use and cognitive difficulties among women compared to men $(\mathrm{p}<0.54)$.

\section{DISCUSSION}

The purpose of this study was to evaluate self-rated cognitive ability in males and females using varying levels of $\mathrm{MJ}$ on the same day in a naturalistic setting. Our findings support previous studies showing that current and heavier MJ use affects self-perceived cognitive ability in both males and females.

These findings support numerous studies showing impairment in cognitive ability in humans following $\mathrm{MJ}$ use $^{17}$, particularly those showing impairments in self-rated every-day memory functioning in MJ users ${ }^{18}$. Such impairment may be related to MJ intoxication, withdrawal, or both. Impairment may also be related in part to the effect of cannabinoids in distinct brain areas such as the hippocampus, an area with the highest density of CB1 receptors that have an important role in cognition ${ }^{19}$. While findings from previous studies have focused on low versus high doses of $\mathrm{MJ}$ or short versus long term effects, our study combines these variables in a naturalistic setting. We found that both men and women reported problems with cognition on a day-to-day basis as a function of daily time spent smoking MJ.

This study utilized minutes of MJ use as a key predictor variable. Since there is no accepted standard of measuring MJ use in the literature, studies have used a wide range of measurement techniques such as number of joints, blunts, bowls, puff volume and duration ${ }^{20}$. However, these measurement techniques can be problematic given the variability of THC content in these methods, and they do not account for different sizes of joints, for example, or situations in which joints are shared or not fully smoked. Although varying amounts of THC can be smoked in similar durations of time, smoking duration quantifies use in a way related to one of the substance use disorder criteria (i.e., time spent on substance-related activities). Therefore, this study may add an additional measure to the literature that is not subject to existing limitations.

This study also used a unique self-perceived measure of cognitive ability, a subcategory of the Medical Outcomes Survey Short-form. This scale has been widely used with wellestablished validity and reliability ${ }^{16,21}$. Use of the cognitive subscale independently of the larger questionnaire may be a clinically useful tool. For example, a patient's self-perceived difficulty with thinking may be identified and highlighted by the clinician as a disadvantage of using MJ.

There were several weaknesses to the current study. First, sleep quantity or quality was not accounted for in this sample. This is important as this young adult sample has a high proportion of sleep problems. An estimated 7.3\% of individuals aged 18-29 meet ICD-10 or DSM-IV criteria for the diagnosis of insomnia ${ }^{22}$. Future studies should analyze sleep and its 
influence on the effects of MJ. Second, tobacco may be used to compensate for experienced deficits or contribute to symptoms if nicotine withdrawal symptoms are experienced. We decided not to include tobacco use as an adjustment in models assessing relationships between MJ use and cognitive difficulty, and therefore, there may have been a potential effect of tobacco use on our findings. Third, we included a limited age range, and these findings may not pertain to adolescents or older adults. Fourth, we did not collect mood measures. Fifth, while we did have a control group of non-users who completed daily assessments of cognition, it is possible that decrements in self-rated cognition may have been confounded by the three week time in the study, i.e. even in the absence of MJ use, the time in the study may have negatively affected subjective or objective cognition. Sixth, some participants reported one or more episodes of heavy drinking during the study, which may have affected self-rated cognitive ability. Seventh, this was a non-laboratory based study and therefore we do not know if MJ use led to deficits in cognition or if deficits in cognition led to MJ use. This question could be explored in future studies by refining the assessment to evaluate the temporal relationship between use and cognitive reporting, or by asking patient to report their understanding of the temporal relationship (e.g. what came first, the marijuana use or the difficulties with thinking?). Last, our recruitment strategies focused on "persons 18-29 who drink alcohol or smoke marijuana." While the sample in the current study did not report significant alcohol use and was selected to represent primary MJ users, this sample may not be as reflective of a typical MJ using population who typically has alcohol-related problems. A recruitment strategy more focused on marijuana only might result in a different population with a greater proportion of MJ only users. One of the study strengths is that approximately half of the study participants in this study were female. Many previous studies on the effect of MJ on cognition have either not included women ${ }^{23,24}$ or included fewer women than men ${ }^{25-30}$.

When sex differences are examined, the vast majority of studies show more impairment in females ${ }^{6,31}$ particularly heavy MJ using females ${ }^{6}$. The explanation for the greater impact in females is likely multifactorial, including physiological, psychological, and psychosocial differences. Some have proposed that the increased fatty tissue stored by women may account for increased storage of $\mathrm{THC}^{31,32}$ and therefore a greater impact on functioning. However, women in our study self-reported similar impairment at all levels of MJ use compared to males, suggesting that marijuana may affect self-reported cognition in females similarly to males. Clinicians and the public should be informed and research should aim to understand this. If replicated in a larger sample, this study suggests that self-rated cognition declines on the day of MJ use. For example, it may be that feeling more cognitively impaired (such as having more ADHD symptoms, doing poorly on exams or other instances of "intellectual testing") may lead to greater marijuana use. Whether this could also be observed in objective testing remains uncertain, but these findings reveal important information regarding one consequence of $\mathrm{MJ}$ use that has real-world meaning to young adult smokers. Whether the use of alcohol and other substances, the duration (e.g. long term use short term versus), frequency of MJ use (e.g. daily versus weekly), and time of assessment relative to last MJ use influence cognitive self-rating is worthy of further study. 


\section{Acknowledgments}

We are grateful for the research support from the National Institute on Drug Abuse (R21 DA031369-01 A1) to Dr. Michael Stein. We are also indebted to the research participants who helped make this research study possible.

\section{References}

1. SAMSA. Substance Abuse and Mental Health Services Administration. Results from the 2012 National Survey on Drug Use and Health: Summary of National Findings. 2013:H-46. HHS. NSDUH Series

2. Lynne-Landsman S, Livingston M, AC W. Effects of State Medical Marijuana Laws on Adolescent Marijuana Use. American Journal of Public Health. 2013; 103:1500-1506. [PubMed: 23763418]

3. Riebe C, Hill M, Lee T, Gorzalka B. Estrogenicregulation of limbic cannabinoid receptor binding. Psychoneuroendocrinology. 2010; 35:1265-1269. [PubMed: 20207489]

4. Rubino T, Parolaro D. Sexually dimorphic effects of cannabinoid compounds on emotion and cognition. Front Behav Neurosci. 2011; 5(64) doi: 10.3389/fnbeh.2011.00064.

5. Marusich J, Lefever T, Antonazzo K, Craft R, Wiley J. Evaluation of sex differences in cannabinoid dependence. Drug Alcohol Depend. 2014; 137:20-28. [PubMed: 24582909]

6. Pope H, Jacobs A, Mialet J, Yurgelun-Todd D, Gruber S. Evidence for sex specific residual effect of cannabis on visuospatial memory. Psychother Psychosom. 1997; 1997(66):179-184. [PubMed: 9259040]

7. Craft R, Marusich J, Wiley J. Sex differences in cannabinoid pharmacology: A reflection of difference in the endocannabinoid system. Life Sciences. 2013; 92:476-481. [PubMed: 22728714]

8. Fattore L, Fratta W. How important are sex differences in cannabinoid action? British Journal of Pharmacology. 2010; 160:544-548. [PubMed: 20590564]

9. Gonzalez R. Acute and non-acute effects of cannabis on brain functioning and neuropsychological performance. Neuropsychology Review. 2007; 17:347-361. [PubMed: 17680367]

10. Hart C, van Gorp W, Haney M, Foltin R, Fischman M. Effects of acute smoked marijuana on complex cognitive performance. Neuropsychopharmacology. 2001; 25:757-765. [PubMed: 11682259]

11. Pope H, Gruber A, Hudson J, Huestis M, Yurgelun-Todd D. Neuropsychological performance in long-term cannabis users. Archives of General Psychiatry. 2001; 58:909-915. [PubMed: 11576028]

12. Pope H, Gruber A, Hudson J, Huestis M, Yurgelun-Todd D. Cognitive measures in long-term cannabis users. Journal of Clinical Pharmacology. 2002; 42:41S-47S. [PubMed: 12412835]

13. Thames A, Arbid N, Sayegh P. Cannabis use and neurocognitive functioning in a non-clinical sample of users. Addict Behav. 2014; 39(5):994-999. [PubMed: 24556155]

14. Becker M, Collins P, Luciana M. Neurocognition in college-aged daily marijuana users. J Clin Exp Neuropsychol. Mar 12.2014 (Ahead of print).

15. First, M.; Spitzer, R.; Gibbon, M.; Williams, J. Structured Clinical Interview for DSM-IV Axis I Disorders (SCID-I), Clinician Version: User's Guide. American Psychiatric Press; Washington, D.C.: 1997.

16. Stewart A, Hays R, Ware J. The MOS short-form general health survey. Reliability and validity in a patient population. Med Care. 1988; 26:724-735. [PubMed: 3393032]

17. Crean R, Crane N, Mason B. An evidence based review of acute and long-term effects of cannabis use on executive cognitive functions. J Addict Med. 2011; 5(1):1-8. [PubMed: 21321675]

18. Rodgers J, Buchanan T, Scholey A, Heffernan T, Ling J, Parrott A. Differential effects of Ecstasy and cannabis on self-reports of memory ability: a web-based study. Hum Psychopharmacol. 2001; 16:619-625. [PubMed: 12404542]

19. Mechoulam R, Parker L. The endocannabinoid system and the brain. Annual Review of Psychology. 2013; 6:21-47.

20. McClure E, Stitzer M, Vandrey R. Characterizing smoking topography of cannabis in heavy users. Psychopharmacology. 2012; 220:309-318. [PubMed: 21922170] 
21. Moorer P, Suurmeijer T. A study of the unidimensionality and cumulativeness of the MOS Shortform General Health Survey. Psychol Rep. 1994; 74:467-470. [PubMed: 8197285]

22. Morin C, LeBlanc M, Daley M, Gregoire J, Merette C. Epidemiology of insomnia: prevalence, self-help treatments, consultations, and determinants of help-seeking behaviors. Sleep Med. 2006; 7:123-130. [PubMed: 16459140]

23. Hunault C, Mensinga T, Böcker K, et al. Cognitive and psychomotor effects in males after smoking a combination of tobacco and cannabis containing up to $69 \mathrm{mg}$ delta-9tetrahydrocannabinol (THC). Psychopharmacology (Berl). 2009; 204(1):85-94. [PubMed: 19099294]

24. Rabin R, Zakzanis K, Daskalakis Z, George T. Effects of cannabis use status on cognitive function, in males with schizophrenia. Psychiatry Res. 2013; 206(2-3):158-165. [PubMed: 23246245]

25. Grant J, Chamberlain S, Schreiber L, Odlaug B. Neuropsychological deficits associated with cannabis use in young adults. Drug Alcohol Depend. 2012; 121:159-162. [PubMed: 21920674]

26. Meier M, Caspi A, Ambler A, et al. Persistent cannabis users show neuropsychological decline from childhood to midlife. Proc Natl Acad Sci. 2012; 109(40):257-264.

27. Secora A, Eddie D, Wyman B, Brooks D, Mariani J, Levin F. A comparison of psychosocial and cognitive functioning between depressed and non-depressed patients with cannabis dependence. $\mathrm{J}$ Addict Dis. 2010; 29(3):325-337. [PubMed: 20635282]

28. Vandrey R, Stitzer M, Mintzer M, Huestis M, Murray J, Lee D. The dose effects of short-term dronabinol (oral THC) maintenance in daily cannabis users. Drug Alcohol Depend. 2013; 128(1-2):64-70. [PubMed: 22921474]

29. Wadsworth E, Moss S, Simpson S, Smith A. Cannabis use, cognitive performance and mood in a sample of workers. J Psychopharmacol. 2006; 20(1):14-23. [PubMed: 16204329]

30. Weinstein A, Brickner O, Lerman $\mathrm{H}$, et al. A study investigating the acute dose-response effects of $13 \mathrm{mg}$ and $17 \mathrm{mg}$ Delta 9- tetrahydrocannabinol on cognitive-motor skills, subjective and autonomic measures in regular users of marijuana. J Psychopharmacol. 2008; 22(4):441-451. [PubMed: 18635724]

31. Anderson B, Rizzo M, Block R, Pearlson G, O’Leary D. Sex, drugs, and cognition: effects of marijuana. J Psychoactive Drugs. 2010; 42:413-424. [PubMed: 21305906]

32. Huestis, M. Pharmacokinetics of THC in inhaled and oral preparations. Humana Press; Totawa, NJ: 1999. 


\section{Table 1}

Demographic characteristics of analysis sample

\begin{tabular}{|c|c|c|c|c|c|c|}
\hline Variable & & Women $(n=22)$ & $\%$ & Men (n=26) & $\%$ & $\mathbf{p}$ \\
\hline Age (sd) & & $21.7(2.68)$ & & $22.8(3.19)$ & & 0.11 \\
\hline Age range & & $18-27$ & & $18-30$ & & \\
\hline \multirow[t]{2}{*}{ Education } & $\leq 12$ & 8 & $44 \%$ & 10 & $55 \%$ & 0.71 \\
\hline & $>12$ & 14 & $50 \%$ & 14 & $50 \%$ & \\
\hline \multirow[t]{2}{*}{ Employment } & Employed & 10 & $46 \%$ & 12 & $55 \%$ & 0.98 \\
\hline & Unemployed & 12 & $50 \%$ & 12 & $50 \%$ & \\
\hline \multirow[t]{2}{*}{ Student } & No & 12 & $44 \%$ & 15 & $56 \%$ & 0.84 \\
\hline & Yes & 10 & $53 \%$ & 9 & $47 \%$ & \\
\hline \multirow[t]{2}{*}{ Tobacco Use } & No & 19 & $51 \%$ & 18 & $49 \%$ & 0.44 \\
\hline & Yes & 3 & $33 \%$ & 6 & $67 \%$ & \\
\hline \multicolumn{7}{|l|}{ Marijuana } \\
\hline \multirow[t]{2}{*}{ Use Disorder } & No & 16 & $49 \%$ & 17 & $52 \%$ & \\
\hline & Yes & 6 & $46 \%$ & 7 & $54 \%$ & 0.99 \\
\hline Marijuana & & & & & & 0.17 \\
\hline Use & No Use & 5 & $56 \%$ & 4 & $44 \%$ & \\
\hline \multicolumn{7}{|l|}{ During Study } \\
\hline \multirow[t]{2}{*}{ Period } & Non-Daily & 10 & $44 \%$ & 13 & $57 \%$ & \\
\hline & Daily & 7 & $44 \%$ & 9 & $56 \%$ & \\
\hline
\end{tabular}

Am J Addict. Author manuscript; available in PMC 2015 April 21. 


\section{Table 2}

Results from generalized negative binomial mixed effects models of daily cognitive self-rating of impairments and minutes of marijuana use among male and female participants.

\begin{tabular}{|c|c|c|c|c|}
\hline Variable & B & Std.Error & t-value & p-value \\
\hline Time (Days & -0.01 & 0.01 & -1.50 & 0.13 \\
\hline Education $\leq 2$ & 0.07 & 0.80 & 0.09 & 0.92 \\
\hline Unemployed & 0.88 & 0.75 & 1.17 & 0.24 \\
\hline Gender: Female & 1.00 & 0.81 & 1.24 & 0.22 \\
\hline Baseline Days of Alcohol Use & -0.01 & 0.07 & -0.20 & 0.84 \\
\hline Minutes of Marijuana Use & 0.004 & 0.001 & 2.76 & 0.006 \\
\hline Gender X Minutes of Marijuana Use ${ }^{a}$ & 0.002 & 0.003 & 0.61 & 0.54 \\
\hline
\end{tabular}

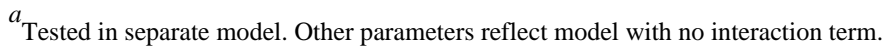

